\title{
Patterns and predictors of nut consumption: results from the 2008/09 New Zealand Adult Nutrition Survey
}

\author{
Rachel C. Brown ${ }^{1 *}$, Siew Ling Tey ${ }^{2}$, Andrew R. Gray ${ }^{3}$, Alexandra Chisholm ${ }^{1}$, Claire Smith ${ }^{1}$, \\ Elizabeth Fleming ${ }^{1}$, Charlie Blakey ${ }^{1}$ and Winsome Parnell ${ }^{1}$ \\ ${ }^{1}$ Department of Human Nutrition, University of Otago, PO Box 56, Dunedin, New Zealand \\ ${ }^{2}$ Clinical Nutrition Research Centre, Singapore Institute for Clinical Sciences, $A^{*}$ STAR, 14 Medical Drive, \\ \#07-02, Singapore 117599, Singapore \\ ${ }^{3}$ Department of Preventive and Social Medicine, University of Otago, PO Box 56, Dunedin, New Zealand
}

(Submitted 11 January 2014 - Final revision received 18 August 2014 - Accepted 5 September 2014 - First published online 30 October 2014)

\begin{abstract}
Regular nut consumption is associated with reduced CVD risk. Insight into nut consumption patterns provides important information to help design strategies to encourage intake. The present study aimed to describe nut consumption in terms of the percentage of consumers, mean grams eaten among the population and nut consumers, and to identify the predictors of nut consumption. Data from the $24 \mathrm{~h}$ dietary recalls of the 2008/09 New Zealand Adult Nutrition Survey ( $n$ 4721) were used to measure nut consumption. On the recall day, the percentages of consumers of whole nuts, nut butters and nuts from hidden sources were 6.9\% ( $n$ 240), 7.2\% ( $n 346)$ and $19 \cdot 2 \%(n 732)$, respectively $(28.9 \%(n$ 1167) combined (total)). The mean grams consumed by the population were relatively low for whole nuts $(2 \cdot 8 \mathrm{~g} / \mathrm{d})$, nut butters $(0.9 \mathrm{~g} / \mathrm{d})$, nuts from hidden sources $(1.5 \mathrm{~g} / \mathrm{d})$ and total nuts $(5.2 \mathrm{~g} / \mathrm{d})$. Among consumers, the mean daily grams of whole nuts, nut butters, nuts from hidden sources and total nuts eaten were $40 \cdot 3,12 \cdot 9,7 \cdot 8$ and $17 \cdot 9 \mathrm{~g} / \mathrm{d}$, respectively. Those aged 15-18 years had the lowest whole nut consumption, but had the highest nut butter consumption. The consumption of total nuts was positively associated with education and socio-economic status, while whole nut consumption was inversely associated with BMI. In conclusion, the low percentage of nut consumers is of concern and new strategies to increase nut consumption are required. Future public health initiatives should be mindful of these patterns and predictors. In particular, different forms of nuts may appeal to different age and socio-economic groups.
\end{abstract}

Key words: Nut consumption: Nut butters: Consumption frequency: Population surveys

Nuts are rich sources of unsaturated fats, protein, fibre and a variety of minerals and vitamins including vitamin $\mathrm{E}$, folate, $\mathrm{Mg}$ and $\mathrm{Ca}^{(1-4)}$. They also contain phytochemicals that along with other nutrients may explain the reduction in chronic disease, in particular CVD, observed in both epidemiological and clinical trials with regular nut consumption ${ }^{(5-9)}$. In addition, although nuts are high in energy, research has suggested that nut consumers have a healthier body weight than those who do not consume nuts ${ }^{(10-14)}$.

The recommendation to regularly consume nuts is an integral component of the dietary guidelines of many countries worldwide. For example, in the USA, a qualified health claim stating that eating $42 \mathrm{~g}$ of nuts per $\mathrm{d}$ may reduce the risk of heart disease was approved in $2003^{(15)}$. In New Zealand, the Heart Foundation guideline recommends a daily consumption of $30 \mathrm{~g}$ of nuts ${ }^{(16)}$
Nut consumption patterns have been described in Europe $^{(17,18)}$ and the USA ${ }^{(19-22)}$. Data from the European Prospective Investigation into Cancer and Nutrition (EPIC) study suggest that there is a clear northern to southern gradient in terms of tree nut consumption ${ }^{(18)}$. For example, in Northern, Central and Southern Europe, the percentage of tree nut consumers has been estimated to be $2.0 \%$ (average intake of $20.3 \mathrm{~g} / \mathrm{d}$ among nut consumers), $4.3 \%$ (average intake of $27 \cdot 2 \mathrm{~g} / \mathrm{d}$ among nut consumers) and $6.3 \%$ (average intake of $29 \cdot 1 \mathrm{~g} / \mathrm{d}$ among nut consumers), respectively. It was thought that this gradient was due to cultural differences, where diets in Southern Europe more closely reflected a Mediterranean-style diet where nuts can provide an important source of energy. Collectively, data from the EPIC study show that the percentage of consumers of total nut including tree nuts, peanuts, nut butters and nuts from hidden sources across ten European

Abbreviations: EPIC, European Prospective Investigation into Cancer and Nutrition; NZ, New Zealand; NZANS, 2008/09 New Zealand Adult Nutrition Survey; NZDep06, New Zealand Index of Deprivation. 
countries was $27 \%$ with a mean gram amount of $14.6 \mathrm{~g} / \mathrm{d}$ among consumers. When considering whole nuts only, the percentage of consumers was $6.9 \%$ with a mean gram amount of $2.2 \mathrm{~g} / \mathrm{d}$ among the whole population and a mean gram amount of $30.8 \mathrm{~g} / \mathrm{d}$ among nut consumers. In Europe, whole tree nut consumption $(4.4 \%)$ was more prevalent than consumption of whole peanuts $(2 \cdot 3 \%)$, with the most popular nuts being walnuts, almonds and hazelnuts, respectively ${ }^{(18)}$. This is in contrast to the USA where data on the annual per capita consumption of nuts, which is based on food disappearance information, suggest that peanuts are more commonly consumed than tree nuts ${ }^{(23)}$. Although there are data on the types of nuts most commonly consumed, to date, studies have not examined the most popular forms of nuts, e.g. raw, roasted and salted. Analysis of data from the 2001-4 National Health and Nutrition Examination Survey (NHANES) showed that the percentage of consumers of any nut (whole nuts, peanut butter, nuts from hidden sources and nuts in recipes) was higher in the USA than in Europe (34 v. 27\%). Interestingly, peanut butter consumption was substantially higher in the USA than in Europe ( $8 v .1 \%)$, whereas consumption of whole nuts was found to be slightly higher in Europe (6.9 v. 6.0\%).

Researchers have also investigated some of the factors associated with nut consumption. In the USA, the percentage of nut consumers among those aged 19-51 years and over 51 years was 5.5 and $8.4 \%$, respectively ${ }^{(20)}$, a statistically significant difference, suggesting that age is associated with nut consumption. Additionally, tree nuts were most likely to be consumed by 'white' adults with higher income and education levels ${ }^{(20)}$. Similar results were found in several European countries, where a higher level of education for the head of the household was associated with higher nut availability $^{(17)}$. In the EPIC cohort, women were more likely to be whole nut consumers, while men who consumed nuts had a higher mean intake ${ }^{(18)}$.

Despite the Heart Foundation recommendation in New Zealand (NZ) to consume $30 \mathrm{~g} / \mathrm{d}$ of nuts, there is no information on the nut consumption patterns of New Zealanders. The 2008/09 New Zealand Adult Nutrition Survey (2008/09 NZANS) provided an opportunity to investigate the percentage of nut consumers and mean grams consumed in the NZ adult population along with the predictors of nut consumption. Furthermore, the 2008/09 NZANS collected information on the types and forms of nuts consumed. Knowledge of nut consumption at a population level will assist in assessing and refining public health initiatives and strategies to encourage regular nut consumption as a means of reducing chronic disease.

\section{Experimental methods \\ Study design and sample}

The 2008/09 NZANS was a cross-sectional survey that used a national sample of NZ adults aged 15 years and older. Participants were recruited using a three-stage selection process involving the selection of 607 mesh blocks using a probability-proportional-to-size design. A mesh block is a small geographical region within NZ as defined by Statistics New Zealand. Each mesh block contains approximately sixty households in rural areas and 110 households in urban areas. A random selection of households within the mesh blocks was followed by a random selection of a participant within the household. Oversampling of Māori and Pacific people, with age groups of 15-18 and 71 years and over, was used in order to achieve adequate numbers for analysis by ethnicity and age. Recruitment took place from late October 2008 to early October 2009. Participation in the survey was voluntary. Before the interview, informed written consent was obtained from each participant, or from the guardian of the participants aged $<18$ years. The present study was conducted according to the guidelines laid down in the Declaration of Helsinki, and all procedures involving human subjects were approved by the New Zealand Health and Disability Multi-Region Ethics Committee (MEC/08/04/049). A full description of the methods and study design is available elsewhere ${ }^{(24)}$.

\section{Dietary assessment}

Survey data were collected at the participant's home by trained interviewers using computer-assisted personal interview software. An interviewer-administered multiplepass $24 \mathrm{~h}$ dietary recall method was used to collect quantitative information on all foods and beverages the participant consumed the previous day (from midnight to midnight) both at and away from home.

In the first stage of the recall, a 'quick list' of all foods, beverages and dietary supplements consumed during the preceding day (midnight to midnight) was obtained. In the second stage, detailed descriptions of all the foods and beverages consumed were collected. Information on any additions made to food before eating was also collected. In the third stage, estimates of the amounts of all foods and beverages consumed were obtained. The amount eaten was described by volume wherever possible (e.g. cups or tablespoons). In addition, food photographs, shape dimensions, food portion assessment aids (e.g. dried beans) and packaging information were used. Finally, in the fourth stage, the foods were reviewed in chronological order and the information collected (including descriptions and amounts eaten) was checked. Repeat interviews were conducted on 1180 participants, but these were not used here.

\section{Determination of nut consumption}

Nut consumption was determined according to the method described by Jenab et al. ${ }^{(18)}$ to allow for comparison with the EPIC study. For the purpose of the analysis, the term 'nuts' includes tree nuts, mixed nuts and peanuts. Chestnuts, coconut and coconut products were not included in the analysis as their nutrient profiles differ from the aforementioned 'nuts'. Tree nuts include almonds, Brazil nuts, cashews, hazelnuts, macadamias, mixed nuts, pecans, pine nuts, pistachios and walnuts.

Nut consumption was assessed using the $24 \mathrm{~h}$ dietary recall data from the ANS08/09 and subsequently divided into three 
categories: (1) whole nuts including tree nuts, mixed nuts and peanuts eaten whole as part of a snack (e.g. mixed nut snacks), or as an addition to a food/meal (e.g. almonds sprinkled on a salad); (2) nut butters including those made from peanuts and tree nuts (e.g. peanut butter and hazelnut spread); (3) nuts from hidden sources including tree nuts, peanuts and mixed nuts eaten as ingredients in recipes or as part of commercial products (e.g. breakfast cereals, snack bars and satay).

The nut type and amounts consumed from hidden sources were determined with the assistance of the 2008/09 NZANS research dietitian who was familiar with $\mathrm{NZ}$ recipes and products. A search for nut ingredients of all generic recipes used in the survey was undertaken. The estimation of nut quantity from these hidden sources is more subject to error; hence, these particular results should be interpreted with greater caution. Total nuts encompass whole nuts, nut butters and nuts from hidden sources.

Participants who reported consuming a non-zero quantity of nuts, of a specific type or overall, in their $24 \mathrm{~h}$ dietary recall were classified as 'nut consumers' of that type or overall. The mean amount of nuts eaten among nut consumers is referred to as 'mean grams eaten among consumers $(\mathrm{g} / \mathrm{d})$ '. The mean amount of nuts eaten by the total population of the 2008/09 NZANS is referred to as 'mean grams eaten among the population $(\mathrm{g} / \mathrm{d})$ '.

\section{Demographic variables}

Demographic variables were selected $a$ priori after reviewing the literature. Variables included sex, age group (15-18, 19-30, 31-50, 51-70 and 71+ years), prioritised ethnicity, New Zealand Index of Deprivation (NZDep06), level of education, BMI and household food security status.

\section{Ethnicity}

Ethnicity was self-reported and participants were categorised into one of three ethnic groups based on a priority classification system using the coding prioritisation order of Māori, Pacific people and New Zealand European and Other.

\section{New Zealand Index of Deprivation}

The NZDep06 is an area-based measure of deprivation that uses nine variables from the NZ census, which reflect specified dimensions of both material and social deprivation. Each mesh block in NZ is given a score between 1 and 10, with a score of 1 reflecting the 'least' deprived areas and 10 the 'most' deprived. For the purpose of the 2008/09 NZANS, these scores were divided into quintiles, where quintile 1 represents the least and quintile 5 represents the most deprived areas.

\section{Education}

Participants reported their highest school level qualification, and, where appropriate, their highest post-school qualification. For the analysis, three groups comprising no formal school qualification, secondary school qualification only or post-school qualification (including trade certificates and university degrees) were derived.

\section{Anthropometry}

Trained interviewers carried out height and weight measurements in duplicate. Standing height was measured using a stadiometer (Seca 214; Seca) and weight using electronic scales (Tanita HD-351; Tanita). BMI was calculated as weight $(\mathrm{kg}) /$ height $(\mathrm{m})^{2}$. The WHO BMI cut-offs were used to categorise BMI status in participants aged 19 years and over. The Cole age- and sex-specific BMI cut-offs were used to categorise BMI status in those aged $15-18$ years $^{(25,26)}$.

\section{Food security}

Household food security was measured using a validated scale based on a series of eight statements developed for use in $\mathrm{NZ}{ }^{(27)}$. The statements were used to assess whether participants considered that their household had a compromised food intake. Participants were classified into three categories, namely full, moderate or low food security, with low representing those who were least food secure.

\section{Statistical analyses}

The complex survey design described above was accounted for in all analyses presented herein. This included incorporating both sampling weights and clustering. The sampling weights used were post-stratified weights for the questionnaire component of the NZANS and based on prioritised ethnicity to reflect population estimates.

Participant characteristics and the percentage of nut consumption are presented as actual frequencies and survey-adjusted percentages. Mean grams of nuts eaten among consumers and the whole population $(\mathrm{g} / \mathrm{d})$ are presented as survey-adjusted means and 95\% CI.

Regression models included sex, age group, prioritised ethnicity, NZDep06 quintile, education, BMI category and food security status, and were used to calculate adjusted (least-squares) means for grams of nuts eaten among consumers. Multiple logistic regression was used to estimate the adjusted OR and 95\% CI, with the aforementioned variables entered into the model, for whole nut, nut butter and total nut consumption. Standard regression diagnostics were used in all cases. Analyses did not include total energy as a covariate because our focus was on absolute amounts, which are consistent with nut consumption guidelines, and identifying groups who might be of interest in public health interventions around nut consumption (including BMI categories). Any associations with total energy or BMI when both were included in a model would be difficult to interpret because of the relationship between these factors.

All statistical analyses were performed using Stata Statistical Software 12.1 (StataCorp LP). All statistical tests were two-sided, and $P<0.05$ was considered statistically significant. For categorical variables, where the overall result was 
statistically significant, pairwise comparisons were made between the levels of that variable. No adjustments for multiple comparisons were made. Tendencies $(0 \cdot 05<P<0 \cdot 10)$ were observed, which may be of interest for future research or suggest the overall patterns.

\section{Results \\ Characteristics of the sample}

Table 1 describes the characteristics of the 2008/09 NZANS sample. A total of 4721 participants were recruited and completed a $24 \mathrm{~h}$ dietary recall.

\section{Percentage of consumers of whole nuts, nut butters,} nuts from hidden sources and total nuts

The percentages of the population consuming whole nuts, nut butters and nuts from hidden sources on the day of the $24 \mathrm{~h}$ dietary recall are shown in Table 2 . The percentage of the population consuming whole nuts was 6.9\%. Among the individual consuming whole tree nuts, the most commonly reported nut intakes were almonds (2.0\%), followed by

Table 1. Characteristics of the survey participants

(Number of participants and survey-weighted percentages)

\begin{tabular}{|c|c|c|}
\hline \multirow[b]{2}{*}{ Demographic } & \multicolumn{2}{|c|}{ Survey participants (all) } \\
\hline & $n$ & $\begin{array}{l}\text { Survey-weighted } \\
\text { percentage }\end{array}$ \\
\hline Total population & 4721 & \\
\hline \multicolumn{3}{|l|}{ Sex } \\
\hline Male & 2066 & 48.6 \\
\hline Female & 2655 & 51.4 \\
\hline \multicolumn{3}{|l|}{ Age (years) } \\
\hline $15-18$ & 699 & $7 \cdot 0$ \\
\hline $19-30$ & 718 & $19 \cdot 7$ \\
\hline $31-50$ & 1344 & $36 \cdot 7$ \\
\hline $51-70$ & 895 & $27 \cdot 1$ \\
\hline $71+$ & 1065 & $9 \cdot 6$ \\
\hline \multicolumn{3}{|l|}{ Ethnicity } \\
\hline NZEO & 2980 & 84.3 \\
\hline Māori & 1040 & $11 \cdot 1$ \\
\hline Pacific & 701 & 4.6 \\
\hline \multicolumn{3}{|l|}{ NZDep06 quintile } \\
\hline Q1 (least deprived) & 664 & $20 \cdot 2$ \\
\hline Q2 & 829 & 21.4 \\
\hline Q3 & 761 & $21 \cdot 3$ \\
\hline Q4 & 1072 & $19 \cdot 0$ \\
\hline Q5 (most deprived) & 1395 & $18 \cdot 1$ \\
\hline \multicolumn{3}{|c|}{ Highest educational qualification } \\
\hline No school qualification & 1217 & $18 \cdot 1$ \\
\hline School & 1413 & $26 \cdot 5$ \\
\hline Post-school & 2057 & 55.4 \\
\hline \multicolumn{3}{|l|}{ BMI $\left(\mathrm{kg} / \mathrm{m}^{2}\right)$} \\
\hline$<25$ & 1409 & 34.9 \\
\hline $25-29 \cdot 9$ & 1581 & $37 \cdot 1$ \\
\hline$\geq 30$ & 1513 & $28 \cdot 0$ \\
\hline \multicolumn{3}{|l|}{ Food security status } \\
\hline Fully & 2454 & $59 \cdot 1$ \\
\hline Moderate & 1693 & 33.7 \\
\hline Low & 488 & $7 \cdot 1$ \\
\hline
\end{tabular}

NZEO, New Zealand European and Other; NZDep06, New Zealand Index of Deprivation. mixed nuts $(1 \cdot 2 \%)$, cashews $(1 \cdot 2 \%)$, Brazil nuts $(0 \cdot 5 \%)$ and walnuts $(0 \cdot 4 \%)$. Consumption of hazelnuts $(<0 \cdot 1 \%)$, macadamias $(0 \cdot 1 \%)$, pecans $(0 \cdot 02 \%)$, pine nuts $(0 \cdot 2 \%)$ and pistachios $(0 \cdot 1 \%)$ were reported by less than $0.3 \%$ of the population. Peanuts were consumed by $1.9 \%$ of the population.

Tree nut butter consumption was only $0.9 \%$, with peanut butter intake being far more prevalent at $6.4 \%$. The percentage of the population consuming nuts from hidden sources was $19 \cdot 2 \%$. When all nut sources were combined, over one-quarter $(28.9 \%)$ of the population consumed nuts on the study day.

There was no evidence for sex differences, although there was a tendency for the percentage of whole tree nut consumption to be higher among females than among males (6.0 v. $4.3 \%, P=0.082)$.

Mean grams of whole nuts, nut butters, nuts from hidden sources and total nuts eaten among the population

Mean grams of tree nuts, peanuts and total whole nuts eaten among the population were $1 \cdot 6,1 \cdot 1$ and $2 \cdot 8 \mathrm{~g} / \mathrm{d}$, respectively. The mean grams of tree nut butters eaten were very low at $0.02 \mathrm{~g} / \mathrm{d}$, whereas the consumption of peanut butter was relatively much higher at $0.9 \mathrm{~g} / \mathrm{d}$. The mean gram of nuts from hidden sources eaten was $1.5 \mathrm{~g} / \mathrm{d}$. When nuts from all sources were considered collectively, the mean gram of total nuts eaten was $5 \cdot 2 \mathrm{~g} / \mathrm{d}$, which is well below the recommended level of $30 \mathrm{~g} / \mathrm{d}$ in NZ.

When examining sex differences, the mean grams of almonds $(P=0.042)$ and 'other nuts' $(P=0.047)$ eaten were significantly higher among females, whereas the mean grams of peanut butter $(P=0.055)$ and nuts from hidden sources $(P=0.065)$ eaten tended to be higher among males.

\section{Mean grams of whole nuts, nut butters, nuts from hidden sources and total nuts eaten among nut consumers}

The mean grams of whole nuts, whole tree nuts and whole peanuts eaten among consumers were 40.3, 31.1 and $58.3 \mathrm{~g} / \mathrm{d}$, respectively (Table 2 ). The mean gram of tree nut butters eaten among consumers was relatively low, namely $2.6 \mathrm{~g} / \mathrm{d}$, whereas the mean gram of peanut butter eaten was $14.0 \mathrm{~g}$. The mean gram of nuts consumed from hidden sources was $7 \cdot 8 \mathrm{~g} / \mathrm{d}$. When nuts from all sources were combined, the gram eaten among all nut consumers was $17 \cdot 9 \mathrm{~g} / \mathrm{d}$.

The mean grams of walnuts, peanut butter, nuts from hidden sources, and combining nuts from all sources eaten were all higher in males than in females (all $P \leq 0.023$ ), whereas the mean gram of 'other nuts' eaten was lower in males $(P=0.035)$.

\section{Predictors of nut consumption}

Tables 3-5 report the adjusted OR (95\% CI) for consuming whole nuts, nut butters and total nuts for a number of demographic variables. There was a tendency for females to be more likely to report total nut consumption than males (OR 1.22, 95\% CI 0.98, 1.50, $P=0.071$ ); however, this 
Table 2. Percentage of nut consumers and mean grams eaten by the population and nut consumers of individual whole tree nuts, peanuts, nut butters, nuts from hidden sources and total sources combined, on the day of the $24 \mathrm{~h}$ recall

(Mean values or percentages and $95 \%$ confidence intervals)

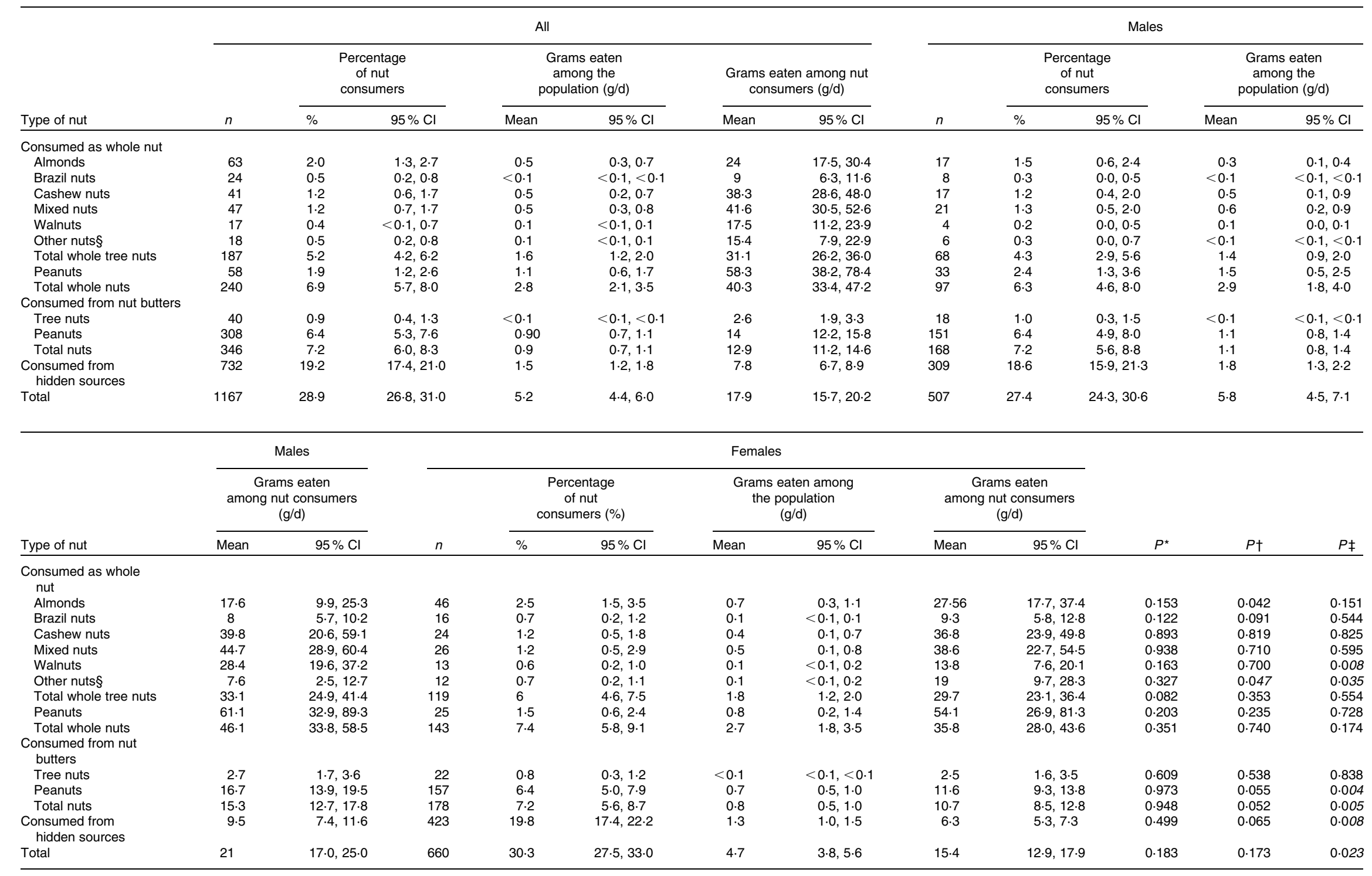

${ }^{*} P$ value for differences in the mean percentage of nut consumers between males and females.

$\dagger P$ value for differences in mean grams eaten among the population $(\mathrm{g} / \mathrm{d})$ between males and females.

$\ddagger P$ value for differences in mean grams eaten $(\mathrm{g} / \mathrm{d})$ among male and female nut consumers.

$\S$ Other nuts consumed by less than ten individuals include hazelnuts, macadamias, pecans, pine nuts and pistachios. 
Table 3. Whole nut consumption in the previous $24 \mathrm{~h}$ and mean grams of whole nuts eaten per $\mathrm{d}$ among consumers (Adjusted odds ratios and $95 \%$ confidence intervals, $n$ 240)

\begin{tabular}{|c|c|c|c|c|c|c|c|c|}
\hline & $\%$ & Adjusted OR* & $95 \% \mathrm{Cl}$ & Adjusted $P \dagger \ddagger$ & $\begin{array}{c}\text { Unadjusted mean grams of } \\
\text { whole nuts eaten among } \\
\text { consumers }(\mathrm{g} / \mathrm{d})\end{array}$ & $\begin{array}{l}\text { Mean adjusted difference in } \\
\text { grams of whole nuts } \\
\text { eaten among consumers ( } \mathrm{g} / \mathrm{d} \text { ) }\end{array}$ & $95 \% \mathrm{Cl}$ & Adjusted $P \nmid \ddagger$ \\
\hline Sex & & & & 0.442 & & & & 0.034 \\
\hline Male & $6 \cdot 3$ & Reference & & & $46 \cdot 1^{a}$ & Reference & & \\
\hline Female & $7 \cdot 4$ & 1.16 & $0.79,1.70$ & & $35 \cdot 8^{\mathrm{b}}$ & -15.0 & $-28 \cdot 8,-1 \cdot 2$ & \\
\hline Age (years) & & & & $<0.001$ & & & & \\
\hline $15-18$ & $1.7^{\mathrm{a}}$ & Reference & & & $38 \cdot 2^{a, b}$ & Reference & & 0.040 \\
\hline $19-30$ & $3 \cdot 6^{a, b}$ & 2.20 & $0.94,5.17$ & & $47 \cdot 6^{a, b}$ & 21.9 & $-15 \cdot 7,59 \cdot 5$ & \\
\hline $31-50$ & $8 \cdot 1^{\mathrm{c}}$ & 4.99 & $2.28,10.90$ & & $37.8^{\mathrm{a}, \mathrm{b}}$ & 0.7 & $-31.0,32.3$ & \\
\hline $51-70$ & $9.4^{\mathrm{c}}$ & 5.99 & $2 \cdot 84,12.66$ & & $44.9^{a}$ & $16 \cdot 2$ & $-18 \cdot 1,50 \cdot 4$ & \\
\hline $71+$ & $6 \cdot 2^{\mathrm{b}, \mathrm{c}}$ & 3.80 & $1.80,8.00$ & & $25 \cdot 3^{\mathrm{b}}$ & -3.8 & $-35.4,27.7$ & \\
\hline Ethnicity & & & & 0.090 & & & & 0.032 \\
\hline NZEO & $7 \cdot 7$ & Reference & & & $40 \cdot 1^{\mathrm{a}}$ & Reference & & \\
\hline Māori & 3.0 & 0.59 & $0.32,1.09$ & & $36 \cdot 3^{\mathrm{a}}$ & -3.5 & $-22 \cdot 0,14 \cdot 9$ & \\
\hline Pacific & 2.2 & 0.47 & $0.21,1.04$ & & $65.8^{\mathrm{b}}$ & 34.7 & $7 \cdot 2,62 \cdot 1$ & \\
\hline NZDep quintile & & & & 0.338 & & & & 0.042 \\
\hline Q1 (least deprived) & 9.8 & Reference & & & $51.5^{\mathrm{a}}$ & Reference & & \\
\hline Q2 & 8.0 & 0.87 & $0.51,1.50$ & & $33 \cdot 5^{b, c}$ & -21.2 & $-40 \cdot 1,-2 \cdot 3$ & \\
\hline Q3 & 6.5 & 0.69 & $0.40,1.19$ & & $29 \cdot 0^{\mathrm{b}}$ & $-26 \cdot 2$ & $-44 \cdot 6,-7 \cdot 7$ & \\
\hline Q4 & $5 \cdot 3$ & 0.60 & $0.34,1.05$ & & $37 \cdot 9^{a, c}$ & -6.5 & $-27 \cdot 7,14 \cdot 7$ & \\
\hline Q5 (most deprived) & 4.5 & 0.65 & $0.36,1.18$ & & $49 \cdot 6^{a, c}$ & 3.1 & $-24.5,30.7$ & \\
\hline Highest educational qualification & & & & 0.274 & & & & 0.055 \\
\hline No school qualification & 4.5 & Reference & & & 28.6 & Reference & & \\
\hline School & 5.8 & 1.36 & $0.77,2.40$ & & 43.5 & $23 \cdot 2$ & $3 \cdot 3,43 \cdot 1$ & \\
\hline Post-school & $8 \cdot 3$ & 1.53 & $0.91,2.56$ & & $41 \cdot 3$ & 14.6 & $-1 \cdot 1,30 \cdot 3$ & \\
\hline BMI $\left(\mathrm{kg} / \mathrm{m}^{2}\right)$ & & & & 0.028 & & & & 0.761 \\
\hline$<25$ & $8 \cdot 7^{\mathrm{a}}$ & Reference & & & 39.1 & Reference & & \\
\hline $25-29.9$ & $6.5^{\mathrm{b}}$ & 0.62 & $0.39,0.97$ & & 42.6 & 0.6 & $-14 \cdot 3,15 \cdot 5$ & \\
\hline$\geq 30$ & $5 \cdot 2^{\mathrm{b}}$ & 0.54 & $0.33,0.89$ & & 39.9 & -4.9 & $-21 \cdot 0,12 \cdot 2$ & \\
\hline Food security status & & & & 0.668 & & & & 0.121 \\
\hline Fully & 8.1 & Reference & & & $42 \cdot 0$ & Reference & & \\
\hline Moderate & $5 \cdot 3$ & 0.81 & $0.39,2.51$ & & 35.7 & $-13 \cdot 2$ & $-27 \cdot 2,0 \cdot 8$ & \\
\hline Low & 5.1 & 1.00 & $0.51,1.29$ & & $40 \cdot 4$ & 9.8 & $-19 \cdot 2,38 \cdot 7$ & \\
\hline
\end{tabular}

NZEO, New Zealand European and Other; NZDep, New Zealand Index of Deprivation

${ }_{\mathrm{a}, \mathrm{b}, \mathrm{c}}$ Values with unlike superscript letters were significantly different $(P<0.05)$

* Calculated using survey logistic regression and adjusted for all other covariates.

$\dagger$ Vverall $P$ value was obtained from regression models.
$\ddagger$ When the overall $P$ values were $<0.05$, pairwise comparisons were made 
Table 4. Nut butter consumption in the previous $24 \mathrm{~h}$ and mean grams of nut butters eaten among consumers

(Adjusted odds ratios and 95\% confidence intervals, $n 346$ )

\begin{tabular}{|c|c|c|c|c|c|c|c|c|}
\hline & $\%$ & Adjusted OR* & $95 \% \mathrm{Cl}$ & Adjusted $P \nmid \ddagger$ & $\begin{array}{l}\text { Unadjusted mean grams of } \\
\text { nut butters eaten among } \\
\text { consumers }(\mathrm{g} / \mathrm{d})\end{array}$ & $\begin{array}{l}\text { Mean adjusted difference in } \\
\text { grams of nut butters } \\
\text { eaten among consumers }(\mathrm{g} / \mathrm{d})\end{array}$ & $95 \% \mathrm{Cl}$ & Adjusted $P \nmid \ddagger$ \\
\hline Sex & & & & 0.993 & & & & 0.001 \\
\hline Male & $7 \cdot 2$ & Reference & & & $15 \cdot 28^{\mathrm{a}}$ & Reference & & \\
\hline Female & $7 \cdot 2$ & 1.00 & $0.72,1.39$ & & $10 \cdot 66^{b}$ & $-5 \cdot 0$ & $-7 \cdot 8,-2 \cdot 1$ & \\
\hline Age (years) & & & & 0.005 & & & & 0.568 \\
\hline $15-18$ & $11 \cdot 7^{\mathrm{a}}$ & Reference & & & $14 \cdot 0$ & Reference & & \\
\hline $19-30$ & $6 \cdot 2^{b, c}$ & 0.54 & $0.30,0.98$ & & $15 \cdot 2$ & 3.4 & $-2 \cdot 8,9 \cdot 6$ & \\
\hline $31-50$ & $9 \cdot 4^{\mathrm{a}}$ & 0.91 & $0.57,1.47$ & & $12 \cdot 4$ & 2.5 & $-2 \cdot 8,7 \cdot 8$ & \\
\hline $51-70$ & $3 \cdot 6^{\mathrm{b}}$ & 0.37 & $0.20,0.69$ & & $12 \cdot 9$ & 4.0 & $-4 \cdot 3,12 \cdot 3$ & \\
\hline $71+$ & $7 \cdot 0^{\mathrm{a}, \mathrm{c}}$ & 0.76 & $0.46,1.27$ & & 9.8 & -0.5 & $-5 \cdot 8,4.9$ & \\
\hline Ethnicity & & & & 0.449 & & & & 0.003 \\
\hline NZEO & $6 \cdot 9$ & Reference & & & $11 \cdot 3^{\mathrm{a}}$ & Reference & & \\
\hline Māori & 9.4 & 1.34 & $0.85,2 \cdot 13$ & & $19 \cdot 2^{\mathrm{b}}$ & 8.0 & $3 \cdot 2,12 \cdot 8$ & \\
\hline Pacific & 7.9 & $1 \cdot 14$ & $0.63,2.04$ & & $19 \cdot 7^{\mathrm{a}, \mathrm{b}}$ & $5 \cdot 1$ & $-1 \cdot 9,12 \cdot 1$ & \\
\hline NZDep quintile & & & & 0.215 & & & & 0.768 \\
\hline Q1 (least deprived) & $5 \cdot 8$ & Reference & & & 9.9 & Reference & & \\
\hline Q2 & $7 \cdot 1$ & 1.28 & $0.72,2.28$ & & 14.0 & 1.8 & $-2 \cdot 3,5 \cdot 9$ & \\
\hline Q3 & 8.5 & 1.49 & $0.81,2.72$ & & 11.9 & 1.3 & $-4 \cdot 4,7 \cdot 1$ & \\
\hline Q4 & 8.9 & 1.64 & $0.95,2.83$ & & 12.9 & -0.2 & $-4 \cdot 7,4 \cdot 3$ & \\
\hline Q5 (most deprived) & 5.5 & 0.93 & $0.51,1.82$ & & 16.5 & 1.6 & $-3.5,6 \cdot 8$ & \\
\hline Highest educational qualification & & & & 0.412 & & & & 0.003 \\
\hline No school qualification & $6 \cdot 4$ & Reference & & & $13 \cdot 8^{\mathrm{a}, \mathrm{b}}$ & Reference & & \\
\hline School & $6 \cdot 8$ & 1.07 & $0.68,1.67$ & & $17 \cdot 9^{a}$ & 2.4 & $-3 \cdot 3,8 \cdot 1$ & \\
\hline Post-school & $7 \cdot 7$ & 1.34 & $0.84,2 \cdot 14$ & & $10 \cdot 5^{\mathrm{b}}$ & $-4 \cdot 6$ & $-10 \cdot 6,1 \cdot 3$ & \\
\hline BMl $\left(\mathrm{kg} / \mathrm{m}^{2}\right)$ & & & & 0.400 & & & & 0.093 \\
\hline$<25$ & $8 \cdot 6$ & Reference & & & $12 \cdot 8$ & Reference & & \\
\hline $25-29 \cdot 9$ & $6 \cdot 7$ & 0.81 & $0.54,1.21$ & & $12 \cdot 3$ & -0.4 & $-4 \cdot 9,4 \cdot 1$ & \\
\hline$\geq 30$ & $6 \cdot 3$ & 0.75 & $0.47,1.20$ & & $13 \cdot 8$ & 0.5 & $-4 \cdot 6,5 \cdot 5$ & \\
\hline Food security status & & & & 0.191 & & & & 0.342 \\
\hline Fully & $6 \cdot 3$ & Reference & & & $12 \cdot 2$ & Reference & & \\
\hline Moderate & $8 \cdot 8$ & 1.36 & $0.92,2.01$ & & $13 \cdot 1$ & -1.7 & $-4 \cdot 9,1 \cdot 5$ & \\
\hline Low & $6 \cdot 0$ & 0.88 & $0.40,1.96$ & & $19 \cdot 0$ & $5 \cdot 8$ & $-4 \cdot 6,16 \cdot 2$ & \\
\hline
\end{tabular}

NZEO, New Zealand European and Other; NZDep, New Zealand Index of Deprivation.

a,b,c Values with unlike superscript letters were significantly different $(P<0.05)$

"Calculated using survey logistic regression and adjusted for all other covariates.

†Overall $P$ value was obtained from regression models.

$\ddagger$ When the overall $P$ values were $<0.05$, pairwise comparisons were made. 
Table 5. Total nut consumption in the previous $24 \mathrm{~h}$ and mean grams of total nuts eaten among consumers

(Odds ratios and $95 \%$ confidence intervals, $n 1167$ )

\begin{tabular}{|c|c|c|c|c|c|c|c|c|}
\hline & $\%$ & Adjusted $\mathrm{OR}^{*}$ & $95 \% \mathrm{Cl}$ & Adjusted $P † \ddagger$ & $\begin{array}{l}\text { Unadjusted mean grams of } \\
\text { total nuts eaten among } \\
\text { consumers }(\mathrm{g} / \mathrm{d})\end{array}$ & $\begin{array}{c}\text { Mean adjusted difference in } \\
\text { total nuts eaten among } \\
\text { consumers }(\mathrm{g} / \mathrm{d})\end{array}$ & $95 \% \mathrm{Cl}$ & Adjusted P†‡ \\
\hline Sex & & & & 0.071 & & & & 0.004 \\
\hline Male & 27.4 & Reference & & & $21 \cdot 0^{\mathrm{a}}$ & Reference & & \\
\hline Female & $30 \cdot 3$ & 1.22 & $0.98,1.50$ & & $15 \cdot 4^{\mathrm{b}}$ & $-6 \cdot 3$ & $-10 \cdot 6,-2 \cdot 0$ & \\
\hline Age (years) & & & & 0.049 & & & & 0.007 \\
\hline $15-18$ & $29 \cdot 2^{a, b}$ & Reference & & & $14 \cdot 1^{\mathrm{a}}$ & Reference & & \\
\hline $19-30$ & $23 \cdot 4^{\mathrm{a}}$ & 0.77 & $0.54,1.08$ & & $18 \cdot 3^{a, b}$ & 6.4 & $-0 \cdot 1,13 \cdot 7$ & \\
\hline $31-50$ & $33.1^{\mathrm{b}}$ & $1 \cdot 19$ & $0.86,1.63$ & & $18 \cdot 2^{\mathrm{b}}$ & $7 \cdot 3$ & $0.5,14.2$ & \\
\hline $51-70$ & $28 \cdot 2^{a, b}$ & 0.97 & $0.71,1.33$ & & $20.5^{\mathrm{b}}$ & 11.1 & $3 \cdot 5,18 \cdot 7$ & \\
\hline $71+$ & $25 \cdot 7^{\mathrm{a}}$ & 0.85 & $0.62,1.17$ & & $11.5^{\mathrm{a}}$ & 1.8 & $-4 \cdot 2,7 \cdot 9$ & \\
\hline Ethnicity & & & & 0.067 & & & & 0.344 \\
\hline NZEO & 30.4 & Reference & & & $17 \cdot 7$ & Reference & & \\
\hline Māori & $22 \cdot 8$ & 0.86 & $0.64,1.15$ & & $18 \cdot 8$ & $2 \cdot 7$ & $-2 \cdot 9,8 \cdot 2$ & \\
\hline Pacific & $17 \cdot 3$ & 0.67 & $0.47,0.94$ & & $22 \cdot 7$ & $5 \cdot 0$ & $-2 \cdot 2,12 \cdot 2$ & \\
\hline NZDep quintile & & & & 0.002 & & & & 0.045 \\
\hline Q1 (least deprived) & $31 \cdot 3^{\mathrm{a}}$ & Reference & & & $23 \cdot 3^{\mathrm{a}, \mathrm{b}}$ & Reference & & \\
\hline Q2 & $32 \cdot 4^{\mathrm{a}}$ & $1 \cdot 11$ & $0.82,1.50$ & & $16 \cdot 2^{\mathrm{a}, \mathrm{b}, \mathrm{c}}$ & -7.4 & $-14.9,0.1$ & \\
\hline Q3 & $34 \cdot 1^{\mathrm{a}}$ & 1.21 & $0.88,1.68$ & & $13 \cdot 9^{c}$ & -9.0 & $-16 \cdot 1,-1 \cdot 8$ & \\
\hline Q4 & $28.1^{\mathrm{a}}$ & 0.96 & $0.69,1.34$ & & $15 \cdot 5^{\mathrm{b}, \mathrm{c}}$ & -7.4 & $-15 \cdot 3,0.4$ & \\
\hline Q5 (most deprived) & $17 \cdot 1^{\mathrm{b}}$ & 0.59 & $0.41,0.85$ & & $24 \cdot 5^{\mathrm{a}}$ & $2 \cdot 0$ & $-8 \cdot 7,12 \cdot 8$ & \\
\hline Highest educational qualification & & & & 0.025 & & & & 0.040 \\
\hline No school qualification & $22 \cdot 0^{\mathrm{a}}$ & Reference & & & $13 \cdot 3^{\mathrm{a}}$ & Reference & & \\
\hline School & $27 \cdot 5^{\mathrm{a}, \mathrm{b}}$ & 1.24 & $0.92,1.65$ & & $20 \cdot 3^{\mathrm{b}}$ & $7 \cdot 7$ & $1 \cdot 7,13 \cdot 6$ & \\
\hline Post-school & $32 \cdot 2^{\mathrm{b}}$ & 1.49 & $1 \cdot 11,2 \cdot 00$ & & $18 \cdot 0^{\mathrm{a}, \mathrm{b}}$ & 3.1 & $-2 \cdot 2,8 \cdot 3$ & \\
\hline BMI $\left(\mathrm{kg} / \mathrm{m}^{2}\right)$ & & & & 0.112 & & & & 0.330 \\
\hline$<25$ & $32 \cdot 6$ & Reference & & & $18 \cdot 8$ & Reference & & \\
\hline $25-29.9$ & $30 \cdot 3$ & 0.89 & $0.69,1.16$ & & $18 \cdot 3$ & -2.4 & $-8.5,3.7$ & \\
\hline$\geq 30$ & 24.5 & 0.73 & $0.55,0.98$ & & $16 \cdot 1$ & -4.6 & $-10 \cdot 7,1.5$ & \\
\hline Food security status & & & & 0.379 & & & & 0.232 \\
\hline Fully & $30 \cdot 6$ & Reference & & & $18 \cdot 3$ & Reference & & \\
\hline Moderate & 28.2 & 1.00 & $0.79,1.28$ & & $16 \cdot 6$ & $-2 \cdot 0$ & $-6 \cdot 9,2 \cdot 8$ & \\
\hline Low & $19 \cdot 0$ & 0.73 & $0.45,1.16$ & & $21 \cdot 6$ & 5.7 & $-4 \cdot 2,15 \cdot 6$ & \\
\hline
\end{tabular}

NZEO, New Zealand European and Other; NZDep, New Zealand Index of Deprivation.

${ }_{a, b, c}$ Values with unlike superscript letters were significantly different $(P<0.05)$

*Calculated using survey logistic regression and adjusted for all other covariates.

$\ddagger$ When the overall $P$ values were $<0.05$, pairwise comparisons were made. 
difference was not apparent for whole nut or nut butter consumption alone.

Associations were found in adjusted models between age and whole nut (overall $P<0 \cdot 001$ ), nut butter (overall $P=0.005$ ) and total nut (overall $P=0.049$ ) consumption. The likelihood of consuming whole nuts was lowest in the 15- to 18-year age group, but nut butter consumption was highest in this age group. When assessing total nut consumption, the highest prevalence was in the 31- to 50-year age group. This age group was significantly more likely to include consumers of total nuts than those aged 19-30 years (OR 1.55, $95 \%$ CI $1 \cdot 11,2 \cdot 16, P=0 \cdot 010$ ) and 71 years and over (OR $1 \cdot 39,95 \%$ CI $1 \cdot 07,1 \cdot 81, P=0 \cdot 015)$.

There were tendencies for the differences between the ethnic groups for consumption of whole nuts (overall $P=0.090$ ) and total nuts (overall $P=0.067$ ), but not for nut butter consumption (overall $P=0.449$ ), with a pattern of lower rates among Māori and Pacific people than among the New Zealand European and Other group for consumption of whole nuts and total nuts.

There was a significant relationship between the percentage of total nut consumption and NZDep06 quintile (overall $P=0.002$ ). Individuals living in the most deprived area (NZDep06-Q5) were significantly less likely to consume total nuts than those living in all the other areas (all $P \leq 0 \cdot 006$ ).

There was a significant relationship between BMI and the percentage of whole nut intake only, with the highest percentage in those with a BMI $<25 \mathrm{~kg} / \mathrm{m}^{2}$ (overall $P=0.028$ ). Both those in the overweight (OR 0.62, 95\% CI 0.39, 0.97, $P=0.036$ ) and obese categories (OR 0.54, $95 \%$ CI 0.33, 0.89, $P=0 \cdot 016)$ were less likely to consume whole nuts compared with those with a healthy BMI. There was no evidence of a difference between the overweight and obese categories ( $P=0 \cdot 600)$.

Education level was a significant predictor of total nut consumption only (overall $P=0 \cdot 025$ ). Individuals with post-school education (OR 1.49, 95\% CI 1·11, 2.00, $P=0 \cdot 007$ ) were significantly more likely to consume nuts than those with no school qualification.

\section{Predictors of grams of nuts eaten}

Tables 3-5 also report the mean grams of nuts eaten per $\mathrm{d}$ among consumers in relation to a number of demographic variables. When adjusting for all other variables in the model, mean grams of whole nuts (Table 3), nut butters (Table 4) and total nuts (Table 5) eaten were significantly higher among males than among females (all $P \leq 0.034$ ).

Overall, there was a significant association between age and mean grams of both whole nuts (overall $P=0 \cdot 040$ ) and total nuts (overall $P=0 \cdot 007$ ) eaten. For whole nuts, pairwise comparisons found higher mean grams eaten among those aged 51-70 years compared with those aged 71 years and over (adjusted difference $20 \cdot 0,95 \% \mathrm{CI} 6 \cdot 2,33 \cdot 8 \mathrm{~g}, P=0 \cdot 004)$. For total nuts, pairwise comparisons found that grams eaten among those aged 31-50 and 51-70 years were significantly higher compared with those aged $15-18$ and 71 years and over.
There was a significant relationship between ethnicity and mean grams of whole nuts (overall $P=0.032$ ) and nut butters (overall $P=0.003$ ) eaten. For whole nuts, Pacific people consumed significantly larger amounts than both New Zealand European and Other (adjusted difference 34.7, 95\% CI 7.2, $62.1 \mathrm{~g}, \quad P=0.013$ ) and Māori (adjusted difference 38.2, $95 \%$ CI 8·3, 68.1 g, $P=0 \cdot 012$ ). For nut butters, Māori consumed significantly greater amounts than the New Zealand European and Other group (adjusted difference $8 \cdot 0,95 \%$ CI 3.2, 12.8 g, $P=0 \cdot 001$ ).

Overall, there was a significant U-shaped relationship between NZDep06 quintile and mean grams of whole nuts (overall $P=0.042$ ) and total nuts (overall $P=0.045$ ) eaten, with the lowest amounts in NZDep06-Q3 and the highest in NZDep06-Q1 and NZDep06-Q5.

In addition, there was a significant relationship between education level and mean grams of nut butters (overall $P=0.003$ ) and total nuts (overall $P=0.040$ ) eaten, with a tendency for whole nuts (overall $P=0.055$ ). For nut butters, pairwise comparisons found that amounts eaten by those with school education was significantly larger than those with post-school education (adjusted difference $7 \cdot 0,95 \% \mathrm{CI}$ $3.0,11.0 \mathrm{~g}, P=0.001)$. For total nuts, the amount consumed by those with school education was significantly larger than those without a school qualification (adjusted difference $7 \cdot 7$, $95 \%$ CI $1.7,13.6 \mathrm{~g}, P=0.012$ ).

\section{Raw and processed nuts}

Whole nut consumption was further categorised into raw, roasted and salted, roasted and unsalted, honey-roasted, and non-specified (Table 6). Overall, $54 \%$ of whole nut consumers ate their nuts raw, 28\% as roasted and salted, $18 \%$ as roasted and unsalted, $3 \%$ as honey-roasted, and $4 \%$ as non-specified (percentages may add to more than $100 \%$ as some nut consumers reported consuming multiple forms). However, tree nuts were more likely to be consumed raw $(67 \%)$ than peanuts (12\%). Only $12 \%$ of tree nut consumers reported consuming as roasted and salted, $19 \%$ as roasted and unsalted, 2\% as honey-roasted, and $6 \%$ as non-specified. In contrast, peanuts were most likely to be consumed as roasted and salted (68\%), followed by roasted and unsalted (18\%), with only $12 \%$ reporting consuming raw peanuts. A further $5 \%$ reported consuming peanuts as honey-roasted.

\section{Discussion}

The present study provides a unique overview of the percentage of nut consumers, and mean grams of whole nuts, nut butters and nuts from hidden sources eaten by the whole population and nut consumers among the NZ population. This provides a valuable baseline for exploring changes in nut consumption in NZ. Furthermore, to our knowledge, this is the first population data on nut consumption in the Southern Hemisphere. Current guidelines in NZ and overseas recommend consumption of $30-42 \mathrm{~g} / \mathrm{d}$ of nuts as a means of reducing CVD risk $^{(15,16)}$. Our data indicate that only $6.9 \%$ of the population consumed whole nuts on the day of their 
Table 6. Percentage of consumers of different forms of tree nuts and peanuts when consumed as whole nuts among the total population

(Mean values and $95 \%$ confidence intervals)

\begin{tabular}{|c|c|c|c|c|c|c|c|}
\hline & \multicolumn{6}{|c|}{ Percentage of consumers } & \multirow[b]{3}{*}{$P \dagger$} \\
\hline & \multicolumn{2}{|c|}{ Total population } & \multicolumn{2}{|c|}{ Males } & \multicolumn{2}{|c|}{ Females } & \\
\hline & Mean* & $95 \% \mathrm{Cl}$ & Mean* & $95 \% \mathrm{Cl}$ & Mean* & $95 \% \mathrm{Cl}$ & \\
\hline Total whole nuts & $6 \cdot 9$ & $5 \cdot 2,8 \cdot 0$ & $6 \cdot 3$ & $4 \cdot 6,8 \cdot 0$ & 7.4 & $5 \cdot 8,9 \cdot 1$ & 0.351 \\
\hline Raw & $3 \cdot 7$ & $2 \cdot 9,4.5$ & $2 \cdot 8$ & $1.7,3.9$ & 4.5 & $3 \cdot 3,5 \cdot 8$ & 0.051 \\
\hline Roasted and salted & 1.9 & $1 \cdot 2,2 \cdot 6$ & $2 \cdot 4$ & $1.2,3.5$ & 1.5 & $0.7,2.3$ & 0.198 \\
\hline Roasted and unsalted & $1 \cdot 2$ & $0.6,1 \cdot 8$ & 0.7 & $0 \cdot 1,1 \cdot 3$ & $1 \cdot 7$ & $0.9,2.5$ & 0.042 \\
\hline Honey roasted & 0.2 & $0.0,0.5$ & 0.4 & $0.0,0.9$ & $<0.1$ & $<0.1,<0.1$ & $<0.001$ \\
\hline Not specified & 0.3 & $0.0,0.6$ & 0.4 & $0.0,1.0$ & 0.2 & $<0.1,0.4$ & 0.310 \\
\hline Total tree nuts & $5 \cdot 2$ & $4 \cdot 2,6 \cdot 2$ & $4 \cdot 3$ & $2 \cdot 9,5 \cdot 6$ & $6 \cdot 0$ & $4 \cdot 6,7 \cdot 5$ & 0.082 \\
\hline Raw & $3 \cdot 5$ & $2 \cdot 7,4 \cdot 2$ & $2 \cdot 6$ & $1 \cdot 6,3 \cdot 7$ & $4 \cdot 2$ & $3 \cdot 0,5 \cdot 4$ & 0.060 \\
\hline Roasted and salted & 0.6 & $<0.1,1.0$ & 0.6 & $0 \cdot 1,1 \cdot 2$ & 0.6 & $0.2,1 \cdot 1$ & 0.986 \\
\hline Roasted and unsalted & 1.0 & $0.5,1.5$ & 0.6 & $0 \cdot 0,1 \cdot 0$ & 1.4 & $0 \cdot 6,2 \cdot 2$ & 0.070 \\
\hline Honey roasted & 0.1 & $0.0,0.3$ & 0.2 & $0.0,0.5$ & $<0.1$ & $<0.1,<0.1$ & 0.055 \\
\hline Not specified & 0.3 & $<0.1,0.6$ & 0.4 & $0.0,1 \cdot 0$ & 0.2 & $<0 \cdot 1,0 \cdot 4$ & 0.310 \\
\hline Total peanuts & 1.9 & $1 \cdot 2,2 \cdot 6$ & $2 \cdot 4$ & $1 \cdot 3,3 \cdot 6$ & 1.5 & $0 \cdot 6,2 \cdot 4$ & 0.203 \\
\hline Raw & 0.2 & $<0.1,0.4$ & 0.2 & $0.0,0.3$ & 0.3 & $0.0,0.7$ & 0.464 \\
\hline Roasted and salted & $1 \cdot 3$ & $0.7,1.9$ & $1 \cdot 7$ & $0 \cdot 7,2 \cdot 7$ & 0.9 & $0.2,1.5$ & 0.161 \\
\hline Roasted and unsalted & 0.3 & $0.1,0.6$ & 0.3 & $0.0,0.7$ & 0.4 & $0.1,0.7$ & 0.784 \\
\hline Honey roasted & 0.1 & $0.0,0.3$ & 0.2 & $0.0,0.6$ & $<0.1$ & $<0 \cdot 1,<0 \cdot 1$ & 0.002 \\
\hline Not specified & 0.0 & & 0.0 & & 0.0 & & \\
\hline
\end{tabular}

* Percentages may add to more than the total as some consumers may have consumed multiple forms within the nut category.

$\dagger P$ value for differences in mean percentage of nut consumers between males and females.

$24 \mathrm{~h}$ dietary recall. This suggests that public health initiatives are needed to increase the prevalence of nut consumption in the NZ population. We identified a number of factors associated with low nut consumption, which should be taken into account when designing strategies to promote nut consumption.

The percentage of whole nut consumers in NZ on their study day was $6.3 \%$ for males and $7 \cdot 4 \%$ for females, which is remarkably similar to that reported in the EPIC study and the 2001-2004 NHANES. The EPIC study reported that the mean percentage of consumers from ten European countries was $6 \cdot 9 \%$, with $6 \cdot 4 \%$ of males and $7 \cdot 2 \%$ of females reporting consumption of whole nuts on the day of the $24 \mathrm{~h}$ dietary recall $^{(18)}$. Comparison with US data is difficult as they define nut consumers differently. Whole nut consumers are termed 'out of hand' nut consumers, defined as individuals who consume nuts solely as nuts and not as part of products, with an intake of at least $7 \mathrm{~g} / \mathrm{d}^{(22)}$. Nevertheless, their percentage of $6 \%$ is similar to that reported in the present study.

In NZ, the most commonly consumed nuts were almonds, peanuts and mixed nuts, which are similar to the USA where the most popular nuts consumed were peanuts, mixed nuts and cashews. However, walnuts, almonds and hazelnuts were the most popular in the EPIC study. The NZ patterns of whole tree nut and peanut consumption (5.2 and 1.9\%) were similar to the EPIC study where the percentages of consumption were 4.4 and $2.3 \%$, respectively; however, peanuts were more commonly consumed than tree nuts in the USA ${ }^{(23)}$.

The mean gram amount eaten among those who consumed whole nuts on the day of the survey was $40 \mathrm{~g} / \mathrm{d}$. This intake is approximately $10 \mathrm{~g} / \mathrm{d}$ higher than European consumers $(30 \cdot 8 \mathrm{~g} / \mathrm{d})$. However, the EPIC study involved ten different countries that had a wide range of intake levels. There was a noticeable north-south gradient, with northern European countries reporting a lower prevalence and quantity of whole nuts consumed. Thus, nut intake patterns in NZ appear to be similar to those in Southern Europe.

In the present study, the percentage of tree nut butter consumption was small (0.9\%), but the percentage of peanut butter consumption was higher at $6.4 \%$, with a mean intake of $14.0 \mathrm{~g} / \mathrm{d}$. The amount is lower than that for whole nuts; however, it does indicate that peanut butter makes a substantial contribution to the nut intake of New Zealanders. Compared with the EPIC cohort, the percentage of peanut butter consumption in NZ was substantially higher $(6.4 \% v$. $1.0 \%)$, and more comparable to the consumption reported in the USA of $8 \%^{(18,20)}$.

Careful attention was placed on identifying the hidden sources of nuts (e.g. cereals, snack bars, satay and confectionery); however, estimating the amount consumed may not be accurate and some food products may have been missed. Therefore, this particular information should be interpreted with greater caution. Nevertheless, the percentage of consumption from these hidden sources (19.2\%) and the average estimated intake among consumers $(7 \cdot 8 \mathrm{~g} / \mathrm{d})$ were again remarkably similar to those reported in the European cohort where nuts from hidden sources were consumed by $20 \cdot 8 \%$ of the population with a mean gram amount of $7.7 \mathrm{~g} / \mathrm{d}$ among consumers. The US percentage of nuts from hidden sources was higher at $25 \%$. This indicates that this nut category makes an important contribution to the nut intake of those in NZ and overseas. However, it should be borne in mind that compared with whole nuts, these sources are more likely to be associated with other ingredients such as 
sugar, added fat and salt. For example, data from the USA reported that of the nuts consumed from hidden sources, confectionery contributed $46 \%$, followed by baked items and desserts (24\%) and cookies (17\%), ahead of ready-toeat cereal (9\%) and meals (4\%).

In line with the data reported in European and US studies, the consumption of whole nuts, nut butters and nuts from hidden sources among nut consumers was higher in males than in females. This is not surprising given the higher energy requirements and intakes of males.

The recommendations for nut consumption are largely based on clinical trials that have investigated the health benefits of raw and unsalted nuts ${ }^{(28,29)}$. The present study is the first large cross-sectional study to investigate which forms (e.g. raw $v$. roasted) of nuts are most commonly consumed. We further categorised whole nuts into raw, roasted, roasted and salted, roasted unsalted, honey-roasted and unspecified. For tree nuts, two-thirds were consumed raw, whereas only $12 \%$ of peanuts were consumed raw. The majority of peanuts (68\%) were consumed as roasted and salted. Females were more likely to consume raw nuts, roasted and unsalted nuts than males. In contrast, males were more likely to consume honey-roasted nuts than females. These sex differences could be due to taste preferences and the context in which nuts were consumed, or may reflect more health-conscious decisions by females. It is unknown whether roasting and flavouring nuts negate some of the positive health benefits observed with raw nut consumption. Indeed, roasting nuts may result in the loss of the skin where many of the antioxidants reside ${ }^{(30,31)}$. These areas warrant further investigation, and the findings would provide important information to inform public health messages.

To promote regular nut consumption, it is important to gain some understanding of the factors associated with nut consumption. In NZ, age and BMI were significant predictors of whole nut consumption, with a tendency for differences between ethnicities. For nut butters, age was the only significant predictor of nut consumption, whereas for total nut consumption, age, NZDep06 and education level were predictors, with ethnicity again showing a tendency.

The percentage of nut consumption was statistically significantly lower in the 15- to 18-year age group compared with those aged over 30 years. Therefore, strategies to increase whole nut consumption in this younger age group may be important. Interestingly, nut butter consumption was highest in this younger age group, and this may be a more preferred source of nuts. To date, there is relatively little information on the health effects of peanut butter. Given this form of nut appears to be important for younger age groups, more research is required to ascertain whether the benefits from raw whole nuts can be extrapolated to nut butters. It is unclear from the present study whether these associations with age reflect either age preferences (which will change over time) or cohort effects.

Cost is one commonly reported barrier to regular nut consumption $^{(32)}$, and these data show that those living in the most deprived areas and with the least education are least likely to consume nuts when all nut sources are combined. This finding is congruent with the results from several European countries ${ }^{(17)}$. Therefore, public health messages need to emphasise the fact that more affordable nuts such as peanuts are also effective in improving diet quality and health status. It is noteworthy that among the groups with the lowest percentage of intake, such as Pacific people and those in the most deprived areas, mean grams of nuts eaten were relatively large. This suggests that public health initiatives may need to emphasise the initiation of nut consumption rather than increasing daily amounts in these groups.

BMI was associated with whole nut consumption. Individuals with a BMI of $<25 \mathrm{~kg} / \mathrm{m}^{2}$ were significantly more likely to consume nuts than those in the overweight and obese categories. The cross-sectional nature of the present study prevents us from determining the direction of this relationship. As nuts are commonly perceived to be fattening, overweight and obese individuals may be less likely to consume them. Conversely, nuts are satiating and, in fact, aid in weight control; therefore, people who consume nuts regularly may be leaner than non-nut consumers. Alternatively, nut consumption may simply be a marker of a healthier diet and thus associated with a healthier body weight. Regardless, these data are in agreement with earlier epidemiological studies indicating that nut consumers tend to be leaner than non-consumers ${ }^{(21,33)}$. Furthermore, several clinical trials that have specifically assessed body weight in response to nut consumption have reported little or no weight gain with the addition of nuts to the regular diet in both lean and overweight individuals ${ }^{(34-38)}$. Mechanisms for the less than predicted weight gain observed in these studies include the satiating effects of nuts, which result in dietary compensation, and the loss of metabolisable energy when whole nuts are consumed ${ }^{(10,13,38,39)}$.

There are some important limitations to bear in mind when interpreting the results of the present study. Dietary data were obtained from a $24 \mathrm{~h}$ recall; thus, many nut consumers will not have consumed nuts on the day of the survey. A limitation with using a single $24 \mathrm{~h}$ dietary recall is that it does not account for day-to-day variations in consumption. Thus, we can only present the likelihood of participants reporting nut consumption on a given day among a representative sample of the NZ population. A further limitation was the identification of hidden sources of nuts. While every attempt was made to identify hidden nuts as ingredients in recipes and commercial food products, the estimation of nut consumption from these sources may have been underestimated.

In summary, the percentage of nut consumers, mean grams eaten among the population and nut consumers in NZ are remarkably similar to those reported in Europe and in the USA. Given the well-documented health benefits of regular nut consumption, the low prevalence of nut consumption reported herein and elsewhere is of concern, and it would appear prudent to adopt strategies to increase nut intake. We have identified potentially important predictors of nut consumption including age, socio-economic status and BMI. Different forms of nuts appear to be more acceptable to different population groups. Further investigation of the 
potential health benefits of different types and forms of nuts is warranted in order to inform public health recommendations. In addition, common barriers to nut consumption such as cost and the perception of weight gain need to be addressed. Combined with the evidence from clinical trials, nuts could be promoted in moderation to those who struggle to maintain a healthy weight given the seemingly low risk of adverse weight gain.

\section{Acknowledgements}

The authors thank all the 4721 New Zealanders who participated in the 2008/09 NZANS.

The 2008/09 NZANS was funded by the New Zealand Ministry of Health. The New Zealand Crown is the owner of the copyright for the survey data. The results presented in this article are the work of the authors.

The authors' contributions were as follows: R. C. B., W. P., A. R. G., E. F. and C. B. designed and/or conducted the research; R. C. B. performed the statistical analyses with assistance from A. R. G.; C. S., E. F. and C. B. managed the dietary analysis; W. P. was the principal investigator of the 2008/09 Zealand Adult Nutrition Survey; R. C. B., S. L. T., A. R. G. and A. C. wrote the manuscript. All authors reviewed and approved the final manuscript.

There are no conflicts of interest.

\section{References}

1. Brufau G, Boatella J \& Rafecas M (2006) Nuts: source of energy and macronutrients. Br J Nutr 96, S24-S28.

2. Ros E \& Mataix J (2006) Fatty acid composition of nuts implications for cardiovascular health. Br J Nutr 96, S29-S35.

3. Salas-Salvado J, Bullo M, Perez-Heras A, et al. (2006) Dietary fibre, nuts and cardiovascular diseases. Br J Nutr 96, S45-S51.

4. Vinson JA \& Cai Y (2012) Nuts, especially walnuts, have both antioxidant quantity and efficacy and exhibit significant potential health benefits. Food Funct 3, 134-140.

5. Afshin A, Micha R, Khatibzadeh S, et al. (2014) Consumption of nuts and legumes and risk of incident ischemic heart disease, stroke, and diabetes: a systematic review and meta-analysis. Am J Clin Nutr 100, 278-288.

6. Bolling BW, Chen CY, McKay DL, et al. (2011) Tree nut phytochemicals: composition, antioxidant capacity, bioactivity, impact factors. A systematic review of almonds, Brazils, cashews, hazelnuts, macadamias, pecans, pine nuts, pistachios and walnuts. Nutr Res Rev 24, 244-275.

7. Luo C, Zhang Y, Ding YS, et al. (2014) Nut consumption and risk of type 2 diabetes, cardiovascular disease, and all-cause mortality: a systematic review and meta-analysis. Am J Clin Nutr 100, 256-269.

8. Ros E (2010) Health benefits of nut consumption. Nutrients 2, 652-682.

9. Zhou DH, Yu HB, He F, et al. (2014) Nut consumption in relation to cardiovascular disease risk and type 2 diabetes: a systematic review and meta-analysis of prospective studies. Am J Clin Nutr 100, 270-277.

10. Flores-Mateo G, Rojas-Rueda D, Basora J, et al. (2013) Nut intake and adiposity: meta-analysis of clinical trials. Am J Clin Nutr 97, 1346-1355.
11. Jackson CL \& Hu FB (2014) Long-term associations of nut consumption with body weight and obesity. Am J Clin Nutr 100, 408S-411S.

12. Mattes RD \& Dreher ML (2010) Nuts and healthy body weight maintenance mechanisms. Asia Pac J Clin Nutr 19, 137-141.

13. Tan SY, Dhillon J \& Mattes RD (2014) A review of the effects of nuts on appetite, food intake, metabolism, and body weight. Am J Clin Nutr 100, 412S-422S.

14. Vadivel V, Kunyanga CN \& Biesalski HK (2012) Health benefits of nut consumption with special reference to body weight control. Nutrition 28, 1089-1097.

15. US Food and Drug Administration (2003) Qualified health claims: letter of enforcement discretion - nuts and coronary heart disease (Docket No. 02P-0505). http://www.fda.gov/ Food/IngredientsPackagingLabeling/LabelingNutrition/ucm 072926.htm

16. Tey S, Brown R \& Chisholm A (2012) Nuts and heart health. In National Heart Foundation of New Zealand Evidence-based Position Statement on the Relationship of Nuts to Heart Health. Auckland: National Heart Foundation of New Zealand.

17. Aranceta J, Rodrigo CP, Naska A, et al. (2006) Nut consumption in Spain and other countries. Br J Nutr 96, S3-S11.

18. Jenab M, Sabat J, Slimani N, et al. (2006) Consumption and portion sizes of tree nuts, peanuts and seeds in the European Prospective Investigation into Cancer and Nutrition (EPIC) cohorts from 10 European countries. Br J Nutr 96, S12-S23.

19. King JC, Blumberg J, Ingwersen L, et al. (2008) Tree nuts and peanuts as components of a healthy diet. J Nutr $\mathbf{1 3 8}$, S1736-S1740.

20. O'Neil CE, Keast DR, Fulgoni VL, et al. (2010) Tree nut consumption improves nutrient intake and diet quality in US adults: an analysis of National Health and Nutrition Examination Survey (NHANES) 1999-2004. Asia Pac J Clin Nutr 19, 142-150.

21. O'Neil CE, Keast DR, Nicklas TA, et al. (2011) Nut consumption is associated with decreased health risk factors for cardiovascular disease and metabolic syndrome in U.S. adults: NHANES 1999-2004. J Am Coll Nutr 30, 502-510.

22. O'Neil CE, Keast DR, Nicklas TA, et al. (2012) Out-of-hand nut consumption is associated with improved nutrient intake and health risk markers in US children and adults: National Health and Nutrition Examination Survey 1999-2004. Nutr Res 32, 185-194.

23. Lino M, Marcoe K, Dinkins JM, et al. (2000) The role of nuts in a healthy diet. Fam Econ Rev 14, 80-82.

24. University of Otago and Ministry of Health (2011) Methodology Report for the 2008/09 New Zealand Adult Nutrition Survey. Wellington: Ministry of Health.

25. Cole TJ, Bellizzi MC, Flegal KM, et al. (2000) Establishing a standard definition for child overweight and obesity worldwide: international survey. BMJ 320, 1240-1243.

26. Cole TJ, Flegal KM, Nicholls D, et al. (2007) Body mass index cut offs to define thinness in children and adolescents: international survey. BMJ 335, 194-197.

27. Parnell W, Wilson N, Mann J, et al. (2005) Overview of food security status in New Zealand as a predictor of nutritional outcomes. Proc Nutr Soc NZ 30, 144-149.

28. Estruch R, Ros E, Salas-Salvado J, et al. (2013) Primary prevention of cardiovascular disease with a Mediterranean diet. N Eng J Med 368, 1279-1290.

29. Sabaté J \& Wien M (2010) Nuts, blood lipids and cardiovascular disease. Asia Pac J Clin Nutr 19, 131-136.

30. Bullo M, Lamuela-Raventos R \& Salas-Salvado J (2011) Mediterranean diet and oxidation: nuts and olive oil as 
important sources of fat and antioxidants. Curr Top Med Chem 11, 1797-1810.

31. Chen C-Y, Milbury PE, Lapsley K, et al. (2005) Flavonoids from almond skins are bioavailable and act synergistically with vitamins $\mathrm{C}$ and $\mathrm{E}$ to enhance hamster and human LDL resistance to oxidation. J Nutr 135, 1366-1373.

32. Pawlak R, Colby S \& Herring J (2009) Beliefs, benefits, barriers, attitude, intake and knowledge about peanuts and tree nuts among WIC participants in eastern North Carolina. Nutr Res Pract 3, 220-225.

33. Bes-Rastrollo M, Sabate J, Gomez-Gracia E, et al. (2007) Nut consumption and weight gain in a Mediterranean cohort: the SUN Study. Obesity 15, 107-116.

34. Fraser GE, Bennett HW, Jaceldo KB, et al. (2002) Effect on body weight of a free 76 kilojoule ( 320 calorie) daily supplement of almonds for six months. J Am Coll Nutr 21, 275-283.
35. Alper CM \& Mattes RD (2002) Effects of chronic peanut consumption on energy balance and hedonics. Int $J$ Obes Relat Metab Disord 26, 1129-1137.

36. Sabate J, Cordero-MacIntyre Z, Siapco G, et al. (2005) Does regular walnut consumption lead to weight gain? Br J Nutr 94, 859-864.

37. Hollis J \& Mattes R (2007) Effect of chronic consumption of almonds on body weight in healthy humans. BrJ Nutr $\mathbf{9 8}$, 651-656.

38. Tey SL, Brown R, Gray A, et al. (2011) Nuts improve diet quality compared to other energy-dense snacks while maintaining body weight. J Nutr Metab 2011, 357350.

39. Novotny JA, Gebauer SK \& Baer DJ (2012) Discrepancy between the Atwater factor predicted and empirically measured energy values of almonds in human diets. $\mathrm{Am} \mathrm{J}$ Clin Nutr 96, 296-301. 